\title{
Stripper foil failure modes and cures at the Oak Ridge Spallation Neutron Source
}

\author{
M. A. Plum, ${ }^{*}$ S. M. Cousineau, J. Galambos, S. H. Kim, P. Ladd, C. F. Luck, C. C. Peters, Y. Polsky, and R. W. Shaw \\ Oak Ridge National Laboratory, Oak Ridge, Tennessee 37831, USA \\ R. J. Macek \\ Los Alamos National Laboratory, Los Alamos, New Mexico 87545, USA \\ D. Raparia \\ Brookhaven National Laboratory, Upton, New York 11973, USA
}

(Received 30 November 2010; published 23 March 2011)

\begin{abstract}
The Oak Ridge Spallation Neutron Source comprises a $1 \mathrm{GeV}, 1.5 \mathrm{MW}$ linear accelerator followed by an accumulator ring and a liquid mercury target. To manage the beam loss caused by the $H^{0}$ excited states created during the $\mathrm{H}^{-}$charge-exchange injection into the accumulator ring, the stripper foil is located inside one of the chicane dipoles. This has some interesting consequences that were not fully appreciated until the beam power reached about $840 \mathrm{~kW}$. One consequence was sudden failure of the stripper foil system due to convoy electrons stripped from the incoming $H^{-}$beam, which circled around to strike the foil bracket and cause bracket failure. Another consequence is that convoy electrons can reflect back up from the electron catcher and strike the foil and bracket. An additional contributor to foil system failure is vacuum breakdown due to the charge developed on the foil by secondary electron emission. In this paper we detail these and other interesting failure mechanisms and describe the improvements we have made to mitigate them.
\end{abstract}

DOI: 10.1103/PhysRevSTAB.14.030102

PACS numbers: 29.20.db, 29.27.Ac, 79.20. $-\mathrm{m}$

\section{INTRODUCTION}

The Spallation Neutron Source accelerator [1] comprises a $1 \mathrm{GeV}, 60 \mathrm{~Hz}, H^{-}$ion beam linac with a 1.5 MW design beam power, followed by an accumulator ring with charge-exchange injection to compress the $1 \mathrm{~ms}$ long pulses from the linac to $\sim 700 \mathrm{~ns}$. The present beam power is typically about $1 \mathrm{MW}$ at $925 \mathrm{MeV}$. Corrugated nanocrystalline diamond stripper foils [2] have been in use since the beginning of formal operations in 2006. These foils were successfully used with no failures until May 3, 2009, shortly after increasing the beam power to $\sim 840 \mathrm{~kW}$. The first failure was quickly followed by two more, and the beam power was reduced to $\sim 430 \mathrm{~kW}$ to prevent further foil system failures, and then to $\sim 400 \mathrm{~kW}$ two days later after another failure. A midcycle foil change (a first for SNS) was executed on May 19, 2009 using a modified foil bracket, but the foil system continued to fail.

A team was assembled to investigate the failures and recommend modifications, which were put in place for the next run cycle that started in September 2009. The modified foils and brackets performed very well, and a

\footnotetext{
*Corresponding author. plum@sns.gov
}

Published by American Physical Society under the terms of the Creative Commons Attribution 3.0 License. Further distribution of this work must maintain attribution to the author(s) and the published article's title, journal citation, and DOI. single foil lasted for the entire September to December production run, which included operation at a beam power of 1 MW. A single foil was also used for the subsequent February to June 2010 run cycle, with even more charge delivered to the neutron production target.

In this paper we discuss the causes of the foil system failures, and the modifications made to prevent them.

\section{SNS STRIPPER FOIL SYSTEM}

The nominally $17 \mathrm{~mm} \times 45 \mathrm{~mm} \times 0.30 \mathrm{mg} / \mathrm{cm}^{2}$ stripper foils have three free edges and are mounted on L-shaped brackets that hang from pins on the foil changing mechanism. The foils are grown on a silicon substrate, and approximately $15 \mathrm{~mm}$ of the top of the substrate is left behind to serve as a handle to support the foil. A photo of a first generation foil and bracket is shown in Fig. 1. The long arm and leg of this bracket style were designed to accommodate stripper foils that require support from thin carbon fibers that can be stretched between the arm and the leg. The diamond foils do not require fiber support.

When in use, the foil is positioned inside a strong $(\sim 0.25 \mathrm{~T})$ magnetic field to control the beam loss caused by the partially stripped $H^{0}$ excited states created by the foil that, if not properly controlled, could strip to $\mathrm{H}^{+}$at some point downstream of the foil and outside the ring acceptance [3], and thus cause excessive beam loss. The magnetic field at the foil causes the excited states with $n \geq 5$ to strip within about a mm of the foil, where $n$ is the principle quantum number of the $H^{0}$ atoms. Also, the foil is 


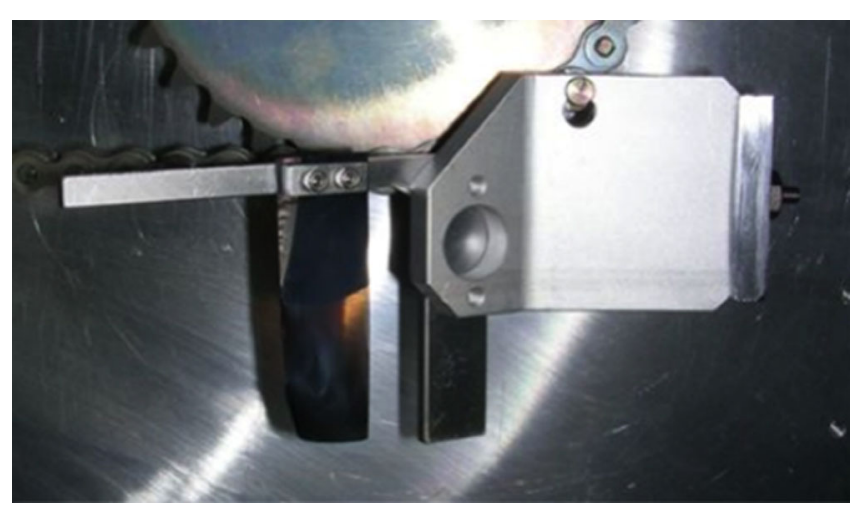

FIG. 1. A used first generation foil and bracket mounted on the foil-changer mechanism. The long arm and leg of the L-shaped bracket were designed to stretch carbon fibers across the span to support foils if needed (not needed in this case). (The figure was reproduced from Ref. [13].)

located in the falling field (downstream end) of the magnet, and the peak field of the next downstream magnet is less than the field at the foil, so that the surviving $n<5$ states will not strip until they reach the secondary stripper foil, whereupon they can be properly transported to a beam dump. The magnetic field causes the $545 \mathrm{keV}$ electrons (often called "convoy" electrons) stripped from the $H^{-}$ beam to circle with a $12 \mathrm{~mm}$ gyroradius. To prevent these electrons from circulating repeatedly through the foil and causing it to overheat, the field is tilted longitudinally by $\sim 200 \mathrm{mrad}$ so that the electron trajectories drop $\sim 16 \mathrm{~mm}$ in the first revolution, which is enough to miss the foil [4].

It is important to properly control the convoy electrons since, e.g., for a $1.5 \mathrm{MW}$ proton beam power there is $1.6 \mathrm{~kW}$ of electron power. A water-cooled electron catcher is mounted to the bottom of the vacuum chamber to intercept the electrons and prevent them from reflecting back up into the path of the beam. The electron catcher comprises carbon-carbon composite wedges that have undercut faces. By design the convoy electrons strike these faces so that any reflected electrons will be aimed downward and away from the path of the proton beam.

\section{FOIL SYSTEM DAMAGE MECHANISMS}

\section{A. Convoy electrons}

The first failure mechanism is simple: the lower portion (leg) of the L-shaped bracket was too close to the foil, so that some of the convoy electrons struck the bracket on their first revolution around the magnetic field lines. It should be stressed that this failure mechanism is actually a bracket failure, not a foil failure. A photograph of a second-generation bracket (the type in use at the time of the first set of failures on May 3, 2009) is shown in Fig. 2. An example of a failed bracket is shown in Fig. 3. The bottom-left corner of the bracket shows the melting that occurred from the convoy electrons striking the bracket.

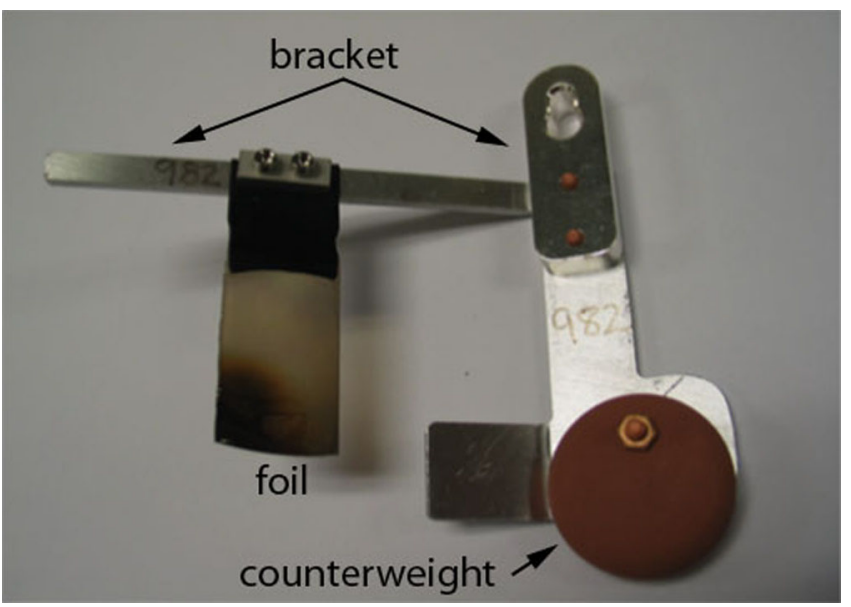

FIG. 2. A used second-generation foil bracket. The beam power in this case was only $400 \mathrm{~kW}$, so this foil and bracket show very little damage. (The figure was reproduced from Ref. [13].)

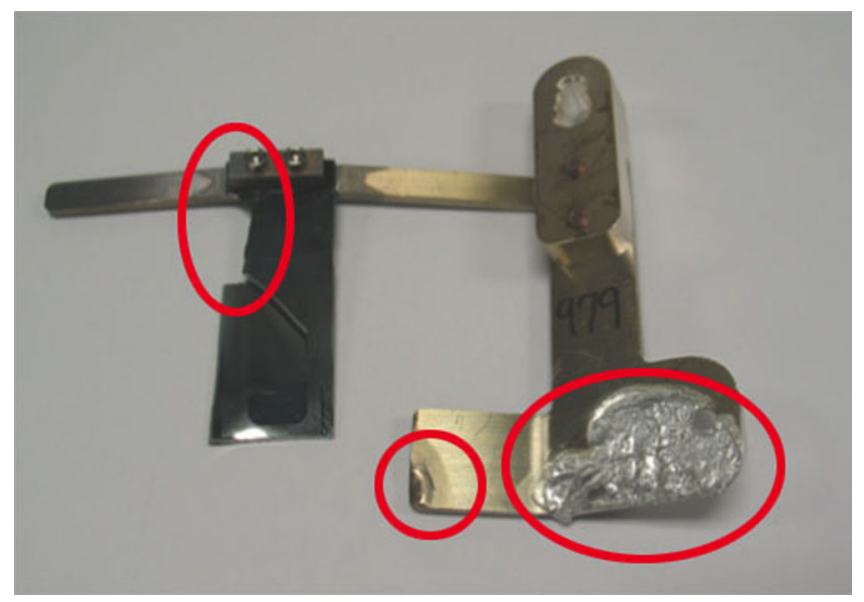

FIG. 3. A failed second-generation foil and bracket. The two lower ellipses indicate the melting damage caused by the electrons. The upper ellipse indicates where the silicon substrate fractured due to forces at the mounting point. This foil lasted for a few hours at $\sim 840 \mathrm{~kW}$ beam power. The convoy electrons struck the bottom-left corner of the tab on the leg of the bracket, and the subsequent heating caused the bracket to melt where the counterweight was attached. (The figure was reproduced from Ref. [13].)

The large melted area on the lower right shows where a circular counterweight was attached before the material around the mounting hole melted, causing the counterweight to fall off. Convoy electron damage can also be seen on the first generation foil brackets, but the beam power was low when these brackets were in use, the damage was minor, and at the time we considered it to be a curiosity. To alleviate this failure mechanism the foil was moved $1 \mathrm{~cm}$ further out on the bracket starting with a portion of the third-generation foils and brackets installed May 19, 2009. 


\section{B. Reflected convoy electrons}

The second failure mechanism is due to reflected convoy electrons. If the electron catcher is not properly positioned relative to the stripper foil, the convoy electrons can miss the undercut faces and instead strike the tops of the wedges, which would make it much more likely for convoy electrons to be reflected back up toward the beam and stripper foil. A $545 \mathrm{keV}$ electron striking a carbon surface at normal angle of incidence has a reflection probability of about $2.5 \%$ [5]. If the electron has a grazing angle of incidence the probability of reflection is higher. For the angles expected in our case the reflection probability is about $40 \%$ [6].

There are several reasons to expect that the electron catcher is not positioned correctly relative to the stripper foil. (1) During ring commissioning in 2006 the beam injection point was moved approximately $7 \mathrm{~mm}$ beam left in order to reduce beam loss caused by unexpected $H^{0}$ and $H^{-}$waste beam trajectories [7]. (2) In 2009 the foil position was moved $1 \mathrm{~cm}$ further out on the bracket arm to increase the separation between the foil and the leg of the bracket (see the previous section). Because the arm is positioned at a $30^{\circ}$ angle relative to the incoming beam, this also moved the foil, and thus the injection point, $5 \mathrm{~mm}$ further downstream. (3) The as-built electron collector position relative to the design injection point is not in accordance with the design.

The electron catcher position was measured on February 8, 2010. Relative to the stripper foil in its original design position, the catcher was found to be $14 \mathrm{~mm}$ too far downstream, $1 \mathrm{~mm}$ too far beam right, and $3 \mathrm{~mm}$ too low. The tolerance on the electron collector position is $\pm 2.5 \mathrm{~mm}$ in the vertical direction, $\pm 6.5 \mathrm{~mm}$ in the longitudinal, and a few $\mathrm{mm}$ in the transverse direction [4], so the as-built position is clearly out of tolerance in the longitudinal direction. The $7 \mathrm{~mm}$ beam left alternate injection point position increases the error in the electron collector position in the transverse direction, and the " $+1 \mathrm{~cm}$ " foil position on the bracket arm reduces the error in the longitudinal direction, but in all cases the electron collector position exceeds the design tolerance.

The trajectories of the reflected convoy electrons were simulated [6] using the PIC-style particle tracking code ORBIT [8], magnetic fields from a detailed 3D model of the magnet [9], and reflection probabilities from an MCNPX [10] simulation. The code transports the electrons though a user-defined step size; interpolates the magnetic field at each step; computes the reflection angle, energy, and direction if relevant; and applies the appropriate momentum kicks. In the model the electron catcher is represented by a flat horizontal carbon plate, but in reality the convoy electrons can strike anywhere on the wedges (or possibly even miss the wedges), so that the reflection can occur over a range of elevations. An example result is shown in Fig. 4, for the case of a third-generation bracket with the

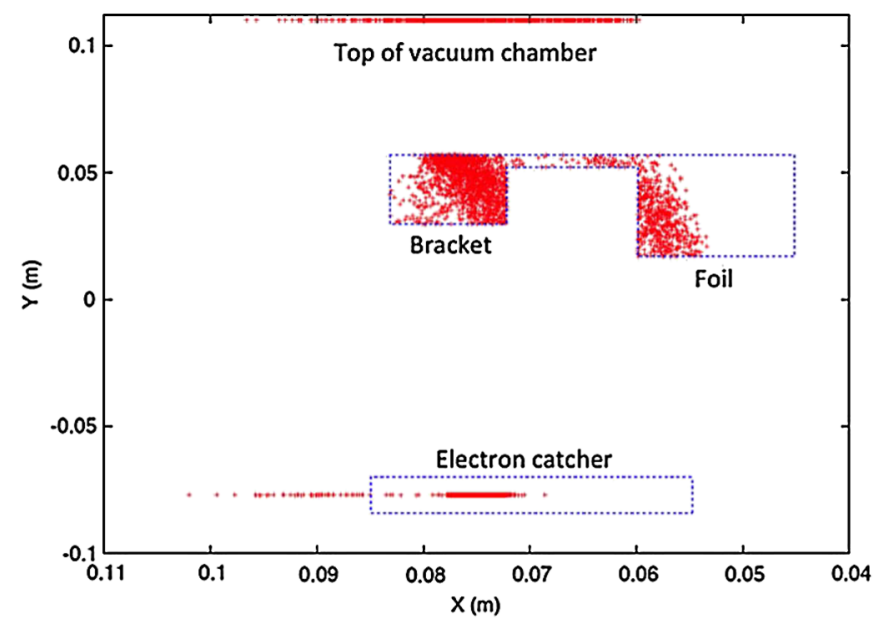

FIG. 4. The results of a particle tracking simulation, for the case of a third-generation foil and bracket, showing the locations where the reflected convoy electrons strike the electron catcher, the stripper foil, the bracket, and the top and bottom of the vacuum chamber.

reflecting surface in the model located halfway up the height of the actual carbon wedges. The reflected electrons can strike the stripper foil, the bracket, the top of the vacuum chamber, or even the bottom of the vacuum chamber (due to magnetic reflections). The model predicts that, for the convoy electrons that do not strike the undercut faces of the electron catcher, (3-17)\% will strike the foil, $(0-1) \%$ will strike the arm of the foil bracket, and (7-25)\% will strike the leg of the foil bracket, where the ranges are calculated by varying the elevation of the reflecting surface from the bottom of the wedges to the top.

Physical evidence for convoy electrons striking the foil bracket can be found, for example, on the third-generation foil bracket shown in Fig. 5. The lower leg of this bracket was removed so that the convoy electrons would not strike it as they travel down to the electron catcher, yet it still shows melting damage on the lower left corner, and the arm of the bracket has also softened enough to allow the arm to droop down. This bracket was used for beam powers up to about $850 \mathrm{~kW}$, and in this case the convoy electron power is $926 \mathrm{~W}$. Based on the simulations discussed above,

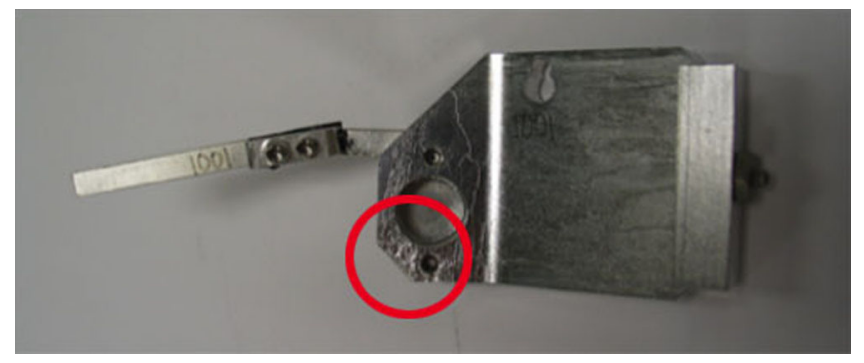

FIG. 5. A used third-generation foil bracket. Melting damage is indicated by the circle. (The figure was reproduced from Ref. [13].) 


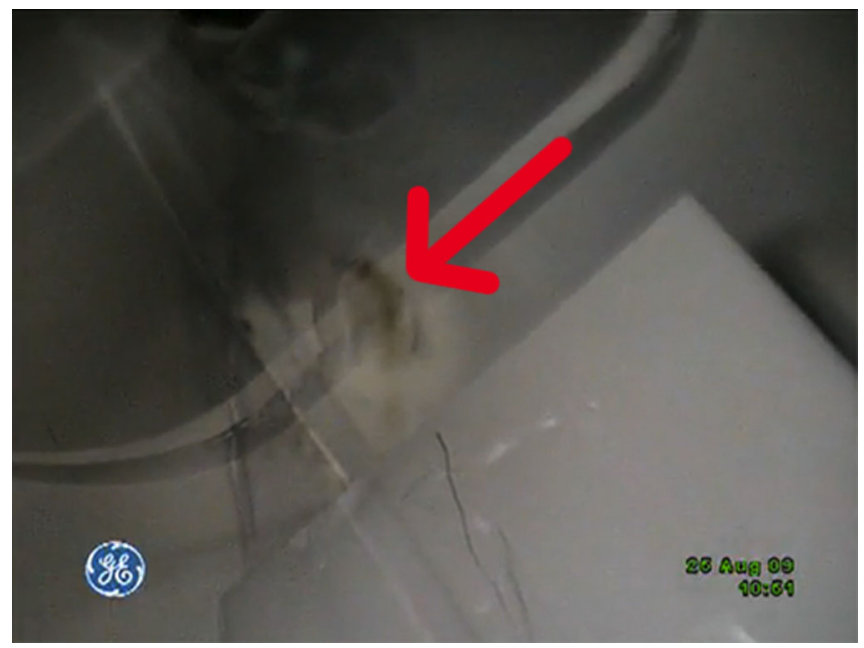

FIG. 6. A photograph of the upper inside surface of the vacuum chamber above the stripper foil. The black ring, indicated by the arrow, is likely due to graphitization caused by reflected convoy electrons.

the power striking the bracket leg could have been as high as $(7-25) \%$ of $926 \mathrm{~W}$, or $65-232 \mathrm{~W}$. We do not have a quantitative measurement of how many convoy electrons are actually caught by the electron catcher and how many are reflected, but finite element analysis simulations predict that just $10 \mathrm{~W}$ directed onto the corner of the bracket is sufficient to cause melting damage.

Further evidence of reflected convoy electrons can be found on the upper surface of the vacuum chamber above the stripper foil. As shown in Fig. 6 there is black ring, likely caused by graphitization from the electrons striking the stainless steel chamber. Black marks are also visible on the tops of the electron collector wedges, presumably due to electron impingement. However, the simulations do not explain all the damage to this bracket. There is also a vertical hole created in the bracket along the inner edge of the foil substrate (see the next section).

\section{Vacuum breakdown}

The third failure mechanism is cathode-spot in-vacuum breakdown [11]. This is a form of electrical breakdown that can take place in a perfect vacuum. To initiate the breakdown, the anode (foil) first develops a positive electrical charge due to secondary electron emission. If the foil is hot enough, thermionic electron emission can further charge the foil. The next step is evaporation of sharp points on the cathode (bracket) that become hot from field emission due to the strong electric field that has been created between the bracket and the foil. The evaporated cathode material then provides the gaseous environment needed to sustain the breakdown. Each breakdown event creates a small crater in the bracket, and over time large holes can develop. Figure 7 shows a close-up photo of the same bracket as in Fig. 5. Several holes can be seen where the silicon foil substrate

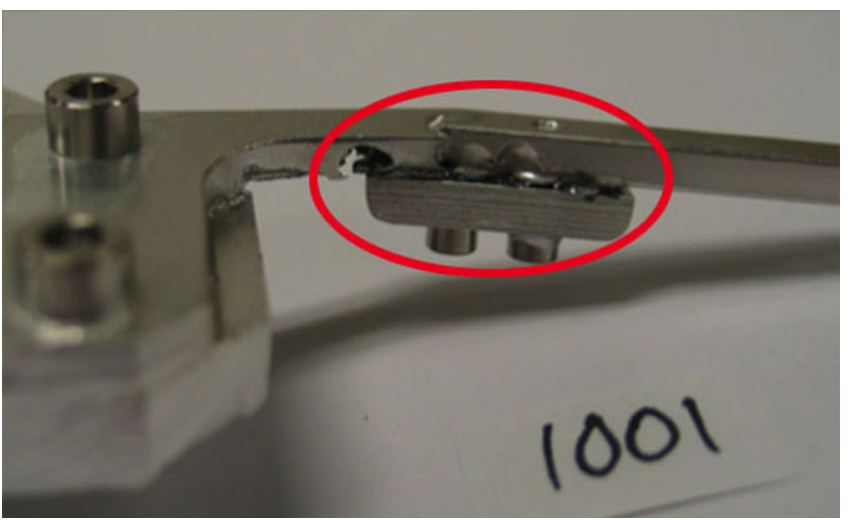

FIG. 7. A close-up view of the foil clamp for the bracket shown in Fig. 5. The ellipse indicates the damage caused by vacuum breakdown. (The figure was reproduced from Ref. [13].)

was clamped to the bracket. One hole passes completely through the bracket arm. The top of the bracket also shows similar material erosion at locations where the foil substrate had sharp edges that helped initiate the vacuum breakdown events.

\section{Bracket pinching}

Even in the absence of reflected convoy electrons and vacuum breakdown, the foil bracket will get hot due to conduction of heat from the irradiated foil. The generations 1 through 3 foil brackets were made of aluminum due to its ease of machining, good conductivity, light weight, and low radioactivation. However, aluminum also has a low melting point and a high coefficient of thermal expansion (CTE) which is approximately 8 times higher than that of the silicon substrate. Titanium screws were used at one point creating an additional CTE mismatch between the fastening components. As the temperature increases in this arrangement, the stripper foil substrate is pinched between the clamp and the bracket arm because the aluminum between the screw head and nut expands more than the

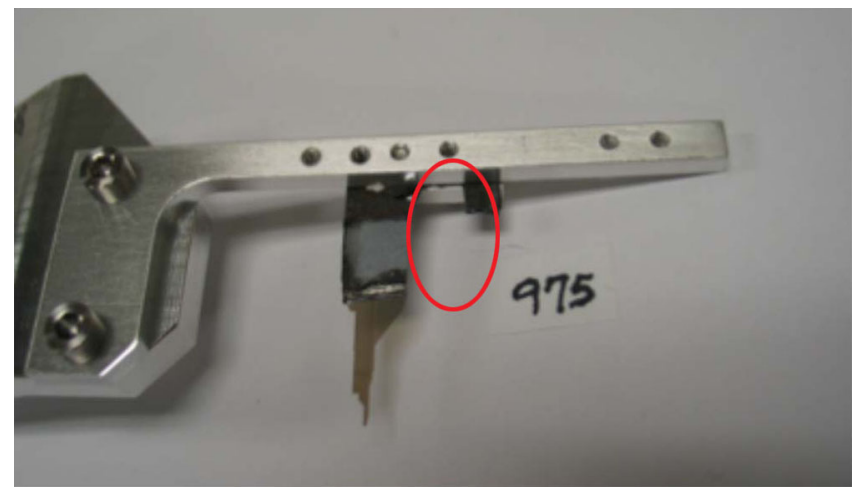

FIG. 8. An example of a fractured foil substrate on a thirdgeneration foil bracket. The ellipse indicates the area where the substrate fractured and pieces fell off. 
titanium. The clamping of the substrate to the holder in combination with the expansion of the holder induces significant tensile and compressive stresses in the silicon substrate. This can cause the silicon to fracture, which can then lead to rips and tears in the freestanding portion of the foil, and also create sharp edges that contribute to the vacuum breakdown. Some of the failed foils exhibited this symptom, such as the one shown in Fig. 8, where most of the right half of the silicon substrate is missing.

\section{E. Other mechanisms}

In addition to the foil system damage mechanisms already discussed, there are others that probably contribute to at least a minor degree. One is circulating beam striking the bracket or the silicon substrate used to mount the diamond foil. The substrate, and some portions of the bracket, are located inside the beam aperture of the ring. Particle tracking simulations do not predict that any particles will be this far away from the closed orbit, yet beam halo is certainly present at a low level (otherwise there would be no beam loss).

Trailing edge multipacting is also likely to be present at some level in the ring, due to the triangular nature of the longitudinal beam profile. In this phenomenon, during the passage of the tail of the proton beam, electrons born at the beam pipe wall are attracted to the center of the proton beam, pass through the beam, and strike the opposite wall with an energy high enough to create more secondary electrons [12]. The energy gain is caused by the decreasing proton beam intensity as the electrons are traveling across the beam pipe. This effect, which is exacerbated by the dipole magnetic field, could cause electrons to strike the foil and bracket and thus cause additional heating and damage.

Sudden beam excursions in the ring, caused by momentary equipment failure, sometimes cause large beam loss in the ring injection area. Some of the beam loss is likely due to beam striking the stripper foil and/or bracket. An example of this type of phenomenon occurred every few minutes and lasted for several days in 2009 due to problems with the ring rf system.

Another heating mechanism that we investigated was eddy current heating due to the pulsed nature of the electric fields of the beam, causing electrical currents to flow in the foil bracket. We have not observed evidence for this type of heating.

\section{SOLUTIONS}

During the summer of 2009 several modifications were made to the foil and the bracket. The bracket material was changed from aluminum to titanium, since the thermal expansion coefficient of titanium is much better matched to that of silicon. Also titanium has a relatively high melting point, good electrical conductivity, and it is lightweight. However, the radioactivation properties are not as good as aluminum due to its higher atomic number. The bracket and clamp were machined flat, and before clamping the foil to the bracket, both the bracket arm and the clamp were carefully polished to remove any sharp points that could contribute to cathode-spot in-vacuum breakdown. Some of the foils we installed in September 2009 were also sandwiched between layers of gold foil $\sim 0.025 \mathrm{~mm}$ thick to help improve the large-area electrical contact between the foil and the bracket. The foils were also moved $1 \mathrm{~cm}$ further out on the arms of the brackets to improve the clearance for the circulating convoy electrons, and the arms and legs of the brackets were made as short as possible to remove any excess material that could be struck by beam halo or reflected convoy electrons. (Note that some additional length would need to be added to both the arm and the leg in order to mount fiber-supported foils.) The foil itself was modified to have a longer freestanding length, increased from $25 \mathrm{~mm}$ to $30-35 \mathrm{~mm}$ (i.e. shorter silicon substrate), to prevent beam halo and reflected convoy electrons from striking the substrate.

A new set of foils, half with the gold foil mounting and half without, were installed for the September to December 2009 run cycle. The first foil selected was one with the gold foil mounting method, and it lasted the entire run cycle, even after increasing the beam power to $1 \mathrm{MW}$. The total charge delivered to the target using this foil was $4820 \mathrm{C}$, to be compared to the previous high-power record of $978 \mathrm{C}$. The used bracket shows no signs of damage. The foil itself is blackened, twisted, and wrinkled, but it was still performing well at the end of the run cycle. A photograph of this foil and bracket, taken after it was removed in February 2010, is shown in Fig. 9.

For the next run cycle, from February to June 2010, we selected a foil mounted without the gold, and that foil also survived the entire run cycle, with an even higher

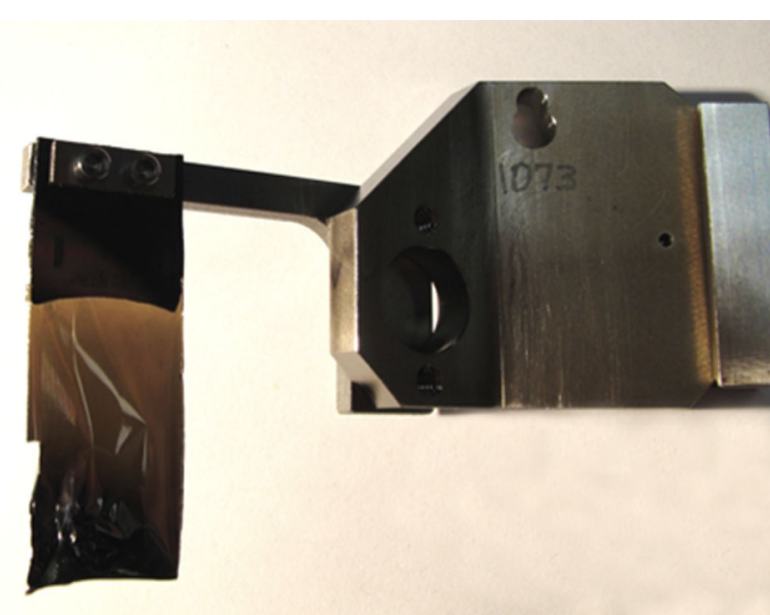

FIG. 9. Photo of the fourth generation foil and bracket that lasted the entire September to December 2009 run cycle at high beam power. (The figure was reproduced from Ref. [13].) 
integrated charge to the target of $7359 \mathrm{C}$. It seems that the gold foil is not necessary. For comparison, at full design beam power, $95 \%$ availability, and 2500 hours per run cycle, the integrated charge to the target would be $12300 \mathrm{C}$.

During beam operation, the real-time signals concerning the stripper foils are limited to the beam loss monitors and the video camera. The only observable difference between the fourth and previous generations of foils and brackets are "hot spots," seen with the video camera, that typically first appear on the bottom edge of the foil, but then later also appear on the surface of the foil near the injected beam spot. These hot spots continue to emit light for several seconds after the beam is shut off-much longer than the approximately one-tenth second needed for the beam spot region to stop emitting light. They are therefore unlikely to be due to simple heating of the foil, especially since some of them lie far away from the region of the foil struck by the core of the beam. We do not have a good explanation for this phenomenon, but one (admittedly questionable) hypothesis is that it may be related to anode-spot in-vacuum breakdown caused by foil charging.

\section{FUTURE PLANS}

The foil lifetime is no longer an issue at the present operating power of $\sim 1 \mathrm{MW}$. However, we are working to continue to ramp up the beam power to the design value of 1.5 MW. We are also working on a beam power upgrade to $3 \mathrm{MW}$ at $1.3 \mathrm{GeV}$. These higher beam powers will place even greater demands on the stripper foil, so we will continue to improve the foils.

To help with the foil charging issue, we are developing more conductive nanocrystalline diamond foils using boron doping. Also, as the foil ages, it tends to develop a curl. We plan to try different corrugation patterns to alleviate this problem. Another issue is that the edge of the foil often has an overhang due to the way the foil is grown on the substrate, where some of the growth occurs on the sides of the substrate rather than just the top. One way to cure this problem is to cut off the edge of the foil prior to etching away the substrate material. The bottom edge of the foil in use at the time of this writing has been cut off.

As we accumulate more experience with this stripper foil technology, we anticipate that we will be able to fabricate stripper foils that will be even better than the ones we have today.

\section{ACKNOWLEDGMENTS}

ORNL is managed by UT-Battelle, LLC, under Contract No. DE-AC05-00OR22725 for the U.S. Department of Energy.

[1] S. Henderson, in Proceedings of the 22nd Particle Accelerator Conference, Albuquerque, New Mexico (IEEE, Piscataway, NJ, 2007), p. 7.

[2] R. Shaw et al., in Proceedings of the 22nd Particle Accelerator Conference, Albuquerque, New Mexico (Ref. [1]), p. 620.

[3] J. Wei et al., Phys. Rev. ST Accel. Beams 3, 080101 (2000).

[4] L. Wang et al., Phys. Rev. ST Accel. Beams 8, 094201 (2005).

[5] G.F. Knoll, Radiation Detection and Measurement (Wiley, New York, 1979), p. 61.

[6] S. Cousineau, in Proceedings of the Advanced Beam Dynamics Workshop on High-Intensity, High-Brightness Hadron Beams, Morschach, Switzerland (2010), http://accelconf.web.cern.ch/AccelConf/HB2010/papers/ mopd41.pdf.

[7] M. Plum, in Proceedings of the Advanced Beam Dynamics Workshop on High-Intensity, High-Brightness Hadron Beams, Nashville, TN (2008), http://accelconf .web.cern.ch/AccelConf/HB2008/papers/wgc04.pdf.

[8] J.A. Holmes, in Proceedings of the First International Particle Accelerator Conference, Kyoto, Japan (2010), p. 1901, http://accelconf.web.cern.ch/AccelConf/IPAC10/ papers/tupec080.pdf.

[9] J. G. Wang, Phys. Rev. ST Accel. Beams 9, 012401 (2006).

[10] MCNPX, https://menpx.lanl.gov/.

[11] A. Anders and G. Y. Yushkov, J. Appl. Phys. 91, 4824 (2002).

[12] R. Macek et al., in Proceedings of the 19th Particle Accelerator Conference, Chicago, Illinois, 2001 (IEEE, Piscataway, NJ, 2001), p. 688.

[13] M. Plum, in Proceedings of the First International Particle Accelerator Conference, Kyoto, Japan, p. 3969, http://accelconf.web.cern.ch/AccelConf/IPAC10/papers/ thpeb039.pdf. 